\title{
Molecular Epidemiology and In Vitro Antifungal Susceptibility of Candida Isolates from Women with Vulvovaginal Candidiasis in Northern Cities of Khuzestan Province, Iran
}

\author{
Shirin Hasanvand, ${ }_{1}^{1}$ Hamid Azadegan Qomi, ${ }^{1}$ Mohammad Kord, ${ }^{2}$ and Mojtaba Didehdar ${ }^{3,}$ \\ ${ }^{1}$ Department of Microbiology, Islamic Azad University, Arak Branch, Arak, Iran \\ ${ }^{2}$ Department of Medical Parasitology and Mycology, Tehran University of Medical Sciences, Tehran, Iran \\ ${ }^{3}$ Infectious Diseases Research Center (IDRC), Department of Medical Parasitology and Mycology, Arak University of Medical Sciences, Arak, Iran \\ "Corresponding author: Mojtaba Didehdar, Department of Medical Parasitology and Mycology, School of Medicine, Arak University of Medical Sciences. P.O. Box: 38195-1884, \\ Arak, Iran. Tel: +98-8614173502, Fax: +98-8634173521, E-mail: didehdar_m@yahoo.com
}

Received 2017 May 08; Accepted 2017 June 24.

\begin{abstract}
Background: Vulvovaginal candidiasis (VVC) is a common infection, affecting up to $75 \%$ of women at least once during their lifetime. In addition, approximately $5 \%$ of patients may experience recurrent VVC. Candida albicans is the most common causative agent of VVC. Overall, precise identification of the causative agents of VVC is necessary for effective treatment.

Objectives: The purpose of this study was to identify the molecular characteristics and antifungal susceptibility of Candida species, isolated from women with VVC in cities of Shoush, Dezful, and Andimeshk, Khuzestan Province, Iran.

Methods: In this descriptive cross sectional study, vaginal samples were collected from 173 women with VVC, referred to gynecologists. The samples were cultured on Sabouraud dextrose agar, containing chloramphenicol. The ITS1-ITS4 region was amplified via polymerase chain reaction (PCR) assay and digested by MspI restriction enzyme. Antifungal susceptibility test was performed for 4 antifungal drugs (fluconazole, nystatin, itraconazole, and clotrimazole) via disk diffusion method.

Results: Out of 173 patients, 95 (54.9\%) showed VVC and 26 (27.4\%) had recurrent VVC. The most common Candida species were C. albicans (70.5\%), C. glabrata (20\%), C. tropicalis (7.4\%), and C. parapsilosis (2.1\%), respectively. The antifungal susceptibility test showed resistance to fluconazole in 1 C. tropicalis, 2 C. albicans, and 3 C. glabrata isolates, while resistance to clotrimazole was detected in $1 C$. albicans and 1 C. glabrata isolate.

Conclusions: According to the results of this study, approximately $30 \%$ of VVC infections were caused by non-C. albicans species, which should be considered by gynecologists due to their azole resistance.
\end{abstract}

Keywords: Antifungal Agents, Candidiasis, Polymerase Chain Reaction, Vulvovaginal, Iran, Candida

\section{Background}

One of the most common infectious diseases of female reproductive organs is vulvovaginal candidiasis (VVC) (1). During reproductive years, almost $75 \%$ of women have at least 1 episode of VVC, while $5 \%$ experience recurrent VVC (RVVC), which is defined as 4 or more episodes of symptomatic VVC over 12 months (2). The signs and symptoms of VVC include itching, thick white discharge, burning sensation, painful intercourse, and vaginal erythema. Although itching and burning sensation are the characteristic symptoms of VVC, they are not adequate to differentiate recurrent diseases from acute ones or determine the appropriate treatment protocol (3).

Candida albicans is the predominant species in VVC (1). Other Candida species, which cause VVC, include $C$. glabrata, C. tropicalis, and C. krusei. The prevalence of these infections is on the rise probably due to the widespread use of antibacterials and azole antifungal drugs (4-9). In general, there are effective drugs for the treatment of VVC. However, drug resistance in Candida species is increasing, and determining the antifungal susceptibility patterns to detect resistant strains and effective treatments is necessary (10-13). Moreover, identification of non-C. albicans species, such as C. glabrata and C. krusei, is important in the treatment of diseases, considering their higher resistance to fluconazole (14).

For identification of Candida species, microscopic methods, culture studies, as well as fermentation and assimilation of sugar content, can be applied. However, traditional methods are sometimes time-consuming and inefficient to identify the involved species $(15,16)$. Nucleic acid-based methods have several advantages over conventional ones, including higher speed, sensitivity, and accuracy; these methods can also identify genera and species in a few hours. On the other hand, the disadvantage of these methods is their high cost, compared with other identification methods (17). Polymerase chain reaction-restriction 
fragment length polymorphism (PCR-RFLP) method is one of the molecular techniques, used for the identification of Candida species (18).

\section{Objectives}

The aim of this study was to identify the molecular characteristics and antifungal susceptibility of Candida species, isolated from women with VVC in Northern cities of Khuzestan province (Shoush, Andimeshk, and Dezful), Iran.

\section{Methods}

\subsection{Ethics Statement}

All patients completed the written informed consent forms, and the study was approved by the deputy of research and ethics committee of Arak University of Medical Sciences (IR.ARAKMU.REC.1395.42).

\subsection{Sample Collection}

A total of 173 women with suspected VVC, referred to gynecology and obstetrics clinics in Northern cities of Khuzestan province (Shoush, Andimeshk, and Dezful), were included in this study.

After studying symptoms such as burning sensation, itching, pain, and cheese-like discharge via physical examination and recording the information in the questionnaire form, samples were collected from vaginal discharge, vaginal posterior fornix, and sides of the vaginal wall, using 2 sterile cotton swabs.

Based on the data, the subjects were within the age range of 14 - 63 years, and the majority of affected patients were in the age group of 21 - 30 years. The clinical symptoms in this study included itching, discharge, and urinary disorders, respectively. The majority of women with VVC were housewives. The most frequent underlying factor for VVC was a history of antibiotic use, followed by pregnancy, diabetes, and use of oral contraceptives.

\subsection{Mycological Examinations}

Using one of the swabs, a section of the specimen was placed on a microscope slide. Then, $20 \%$ potassium hydroxide $(\mathrm{KOH})$ was added and examined under a light microscope for yeast cells and pseudomycelium. The other swab was inoculated on Sabouraud dextrose agar (Merck, Germany), containing chloramphenicol, and incubated for 48 - 72 hours at $28-30^{\circ} \mathrm{C}$. Colonies growing first were used for microscopy via methylene blue staining. Then, they were subcultured on CHROMagar ${ }^{\mathrm{TM}}$ Candida medium (CHROMagar, France) and incubated at $35^{\circ} \mathrm{C}$ for 48 hours.
Differentiation of Candida species from other clinically important yeast species was performed according to different colors produced by Candida colonies in the chromogenic medium, based on the manufacturer's protocol.

\subsection{Molecular Identification}

\subsubsection{DNA Extraction}

Fungal genomic DNA was extracted from each isolate colony, according to a previously described method (19). Briefly, about $5 \mathrm{~mm}^{3}$ of cultured yeast cells was transferred to a $1.5 \mathrm{~mL}$ microcentrifuge tube, and $300-\mathrm{mg}$ glass beads (0.5 mm diameter), $300 \mu \mathrm{L}$ of lysis buffer (200 mM tris-Hcl, pH: 8; 25 mM EDTA; 250 mM NaCl; and 0.5\% sodium dodecyl sulfate), and $300 \mu \mathrm{L}$ of phenol chloroform-isoamyl alcohol were added.

The samples were shaken vigorously for 5 minutes and centrifuged for 5 minutes at $10000 \mathrm{rpm}$. The supernatant was then transferred to a fresh tube and extracted again with chloroform. Then, the same volume of isopropanol and $3 \mathrm{M}$ sodium acetate (volume, $0.1 ; \mathrm{pH}, 5.2$ ) were added. The solution was then vortexed, incubated for 10 minutes at $-20^{\circ} \mathrm{C}$, and centrifuged for 15 minutes at $12000 \mathrm{rpm}$. The precipitants were washed with ice-cold 70\% ethanol, dried in air, dissolved in $50 \mu \mathrm{L}$ of distilled water, and stored at $20^{\circ} \mathrm{C}$.

\subsubsection{PCR-RFLP Analysis}

All clinical isolates were assessed using PCR-RFLP assay, as described in the literature (20). Briefly, the internal transcribed spacer (ITS) rDNA region was amplified using universal primers, ITS1 (5'-TCCGTAGGTGAACCTGCGG3') and ITS4 (5'-TCCTCCGCTTATTGATATGC-3') (21). The amplified products were digested with MSPI (FastDigest, Fermentas life sciences, Lithuania) at $37^{\circ} \mathrm{C}$ for 10 minutes (22). The PCR amplicons and digested products were respectively electrophoresed on $1 \%$ and $2 \%$ agarose gel in tris/borate/EDTA (TBE) buffer and stained with ethidium bromide $(0.5 \mu \mathrm{g} / \mathrm{mL})$. For identification of the isolates, RFLP patterns were compared with the standard profiles.

\subsection{In Vitro Antifungal Susceptibility Testing}

Antifungal susceptibility to antifungal disks (Liofilchem, Italy), including fluconazole (25 $\mu \mathrm{g})$, nystatin (100 IU), itraconazole (50 $\mu \mathrm{g}$ ), and clotrimazole (50 $\mu \mathrm{g}$ ), was analyzed for Candida isolates using disk diffusion method, according to the clinical and laboratory standards institute (CLSI) guidelines (23). Briefly, MuellerHinton Agar (Merck, Germany) was prepared with 2\% glucose and $0.5 \mu \mathrm{g} / \mathrm{mL}$ of methylene blue.

After adjusting the turbidity of the inoculum suspension of yeast cells to 0.5 McFarland standard, a sterile cotton swab was dipped into the adjusted suspension, rotated 
several times, and inoculated on the agar plate. Antifungal disks were placed on the agar surface after 5 to 15 minutes. Then, the plates were incubated for 24 hours at $35^{\circ} \mathrm{C}$. After incubation, the zone diameters surrounding the disks were measured. Susceptibility to the tested drugs, which was categorized as susceptible (S), intermediate susceptible (I), and resistant (R), was in accordance with the manufacturer's guidelines.

\section{Results}

In a total of 173 women with VVC, 95 (54.9\%) were positive on direct examination. All these patients were positive for Candida growth in culture and were infected with 1 Candida species. Among patients with VVC in this study, 33 (34.7\%) had 1 episode of vaginal infection, 26 (27.4\%) had experienced several episodes of infection, and 36 (37.9\%) had no record of infection (Table 1 ).

The PCR assay with universal primers, ITS1 and ITS4, was performed, presenting different sizes of products (510 - 871 bp), as expected, depending on the Candida species (Figure 1). The obtained RFLP pattern for each isolate is shown in Figure 1. The prevalence of different Candida species with RFLP is as follows: C. albicans (70.5\%), C. glabrata (20\%), C. tropicalis (7.4\%), and C.parapsilosis (2.1\%) (Table 1 ).

In antifungal susceptibility testing, among 95 detected Candida species, 6 were resistant to fluconazole, (2 C. albicans, 3 C. glabrata, and 1 C. tropicalis isolate). In addition, clotrimazole resistance was observed in 2 patients ( 1 C. albicans and 1 C. glabrata isolate). Generally, 8 cases of drug resistance were reported in this study (Table 2); all drugresistant strains were isolated from women with RVVC.

\section{Discussion}

In the present study, $54.9 \%$ of the patients showed VVC. The infection was more prevalent in the age group of 20 40 years, which is in agreement with the majority of previous studies performed in Iran (24-28). Vulvovaginal candidiasis is more prevalent in this age group probably due to the excessive use of contraceptive methods, such as intrauterine devices and oral contraceptive pills. In agreement with previous studies in Iran and other countries (2, 24, 25, 29-31), C. albicans was the most common pathogen isolated from VVC patients in the present study. However, in a study by Mohanty et al. (32), C. glabrata was the main species isolated from VVC patients. The present finding could be related to the greater ability of C. albicans in adhesion to the vaginal mucosa. Candida glabrata was the second most common species in the present study, which is consistent with the findings of some reports from Iran and other countries $(24,27,28,33-35)$.
Moreover, other non-C. albicans species, including $C$. tropicalis (7.4\%) and C. parapsilosis (2.1\%), were detected in the present study. Due to the excessive use of azole drugs and poor response of some non-C. albicans species (especially C. glabrata) to these drugs (eg, fluconazole), the prevalence of non-C. albicans species has increased in VVC patients (35). Moreover, in line with previous reports from Iran and different countries $(2,24,25,28,36,37)$, mixed Candida species were not isolated in the present study.

In the current research, $29.5 \%$ of Candida isolates were non-C. albicans species, while in a study by Mahmoudabadi et al. (38) in Ahvaz, Iran, the prevalence of pathogenic species was only 6\%. Similar to our study, Roudbari et al. (36) identified C. albicans, C. glabrata, C. tropicalis, and C. parapsilosis isolates in VVC patients. Additionally, in the present study, according to the clinical examinations by gynecologists and previous experimental findings, 27.3\% of the patients were affected by RVVC. In agreement with the present study, Richter et al. (35) evaluated 593 vaginal Candida isolates and found C. albicans and C. glabrata to be the most common species in RVVC patients. The high frequency of $C$. albicans isolates from RVVC patients could be attributed to the importance of host factors versus pathogenic factors (26).

In the present study, the antifungal susceptibility results showed that all Candida species were susceptible to nystatin and itraconazole, while $97.9 \%$ and $93.7 \%$ were susceptible to clotrimazole and fluconazole, respectively. In the current study, the highest frequency of resistance to azole drugs (fluconazole and clotrimazole) was observed in C. glabrata (4.2\%), followed by C. albicans (3.1\%) and C. tropicalis (1\%). Consistent with other previous studies (27,35), C. glabrata isolates were the most resistant to fluconazole. In a study performed in Ahvaz (38), none of Candida isolates were resistant to clotrimazole, while in our study, clotrimazole resistance was observed in C. albicans and C. glabrata isolates.

In contrast to some studies in different countries (3942), $2.1 \%$ of C. albicans isolates were resistant to fluconazole in the present research. Similar to studies by Fan et al. (2) and Richard et al. (35), high susceptibility of Candida isolates was reported in the current study, which could be related to the widespread use of azoles in VVC patients in this region of Iran. As our findings showed, all resistant species to azoles were isolated from RVVC patients. Since these species were susceptible to nystatin, this drug could be an option in the treatment of RVVC patients.

\subsection{Conclusion}

In conclusion, C. albicans is still the most common Candida species, isolated from VVC patients. Since the majority 
Table 1. Distribution of Patients with Vulvovaginal Candidiasis (VVC) and Recurrent VVC (RVVC) Based on the Isolated Candida Species

\begin{tabular}{|c|c|c|c|}
\hline Species & Number of Patients with VVC (\%) & Number of Patients with RVVC (\%) & Total (\%) \\
\hline C. albicans & $48(50.5)$ & $19(20)$ & $67(70.5)$ \\
\hline C.glabrata & $14(15.8)$ & $5(4.2)$ & $19(20)$ \\
\hline C. tropicalis & $5(4.2)$ & $2(9.5)$ & $7(7.4)$ \\
\hline C. parapsilosis & $2(2.1)$ & - & $2(2.1)$ \\
\hline Total (\%) & $69(72.7)$ & $26(27.3)$ & $95(100)$ \\
\hline
\end{tabular}

Figure 1. Representation of PCR-RFLP Assay on Candida Isolates

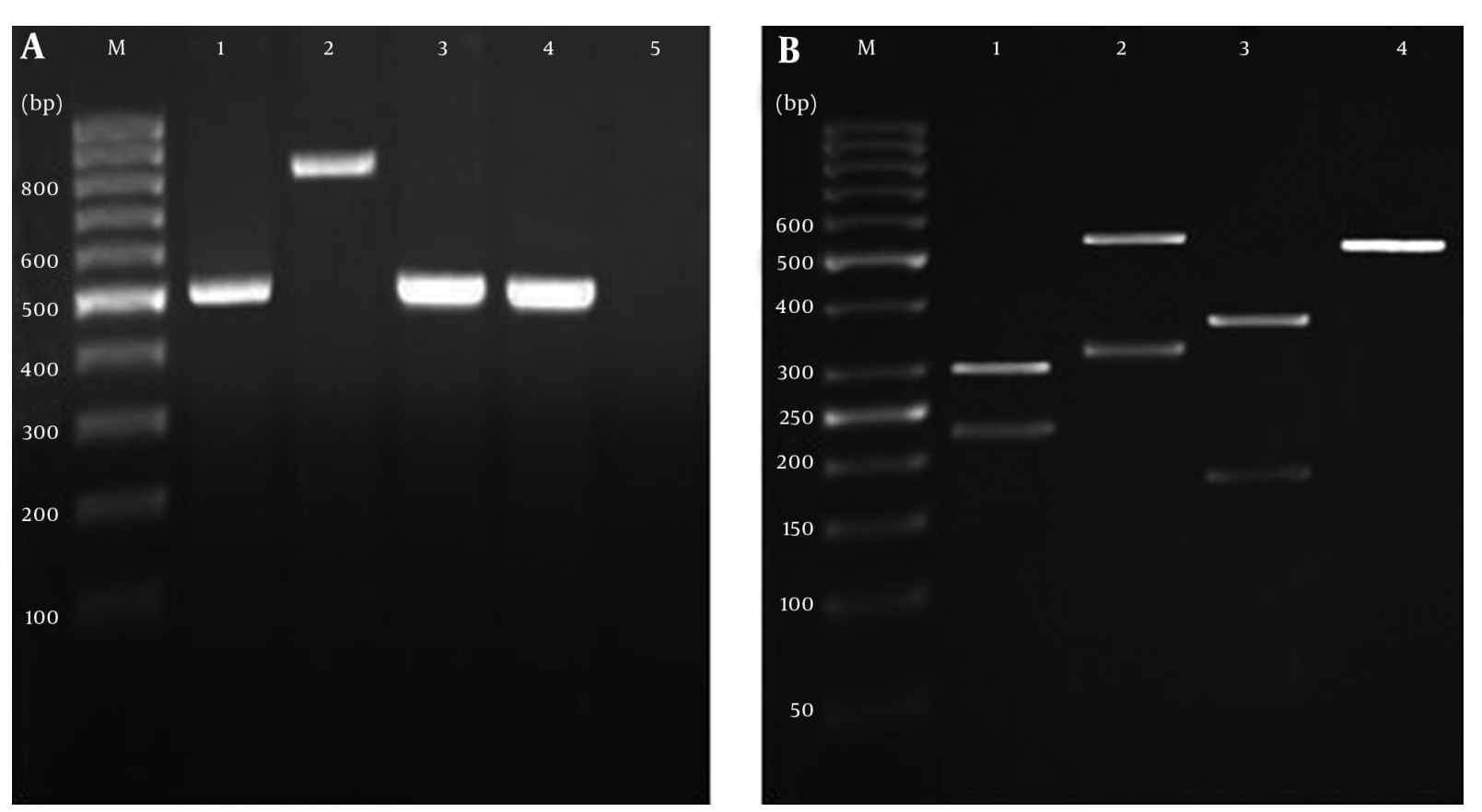

A) PCR products before the digestion step, B) profiles of ITS-RFLP products after digestion using MSPI enzyme (lane 1, C. albicans; lane 2, C. glabrata; lane 3, C. tropicalis; lane 4, C. parapsilosis; lane 5, negative control; lane M, DNA size marker)

Table 2. Antifungal Drug Resistance of Candida Isolates in the Present Study

\begin{tabular}{lccc}
\hline Species & C.albicans & C.glabrata & C.tropicalis \\
\hline Fluconazole & 2 & 3 & 1 \\
Clotrimazole & 1 & 1 & 0 \\
Nystatin & 0 & 0 & 0 \\
Itraconazole & 0 & 0 & 0 \\
\hline
\end{tabular}

of resistant isolates to azoles are non-C. albicans species, gynecologists are recommended to use a suitable drug, such as nystatin, for the treatment of RVVC.

\section{Acknowledgments}

The authors would like to thank the participants for their cooperation, without which we could not complete this study. 


\section{Footnotes}

Authors' Contribution: Mojtaba Didehdar: study concept, study design, interpretation of data, revision of the manuscript, and study supervision; Hamid Azadegan Qomi: phonotypical and molecular analyses; Shirin Hasanvand and Mohammad Kord: acquisition of data and statistical analysis; all authors read and approved the final manuscript.

Conflicts of Interest: The authors declare no conflicts of interest regarding the publication of this paper.

Financial Disclosure: There is no financial disclosure to report.

Funding/Support: This study was supported by Islamic Azad University of Arak, Arak, Iran (university ethics committee code, 1395.42).

\section{References}

1. Sobel JD. Vulvovaginal candidosis. Lancet. 2007;369(9577):1961-71. doi: 10.1016/S0140-6736(07)60917-9. [PubMed: 17560449].

2. Fan SR, Bai FY, Liao QP, Liu ZH, Li J, Liu XP. Genotype distribution of Candida albicans strains associated with different conditions of vulvovaginal candidiasis, as revealed by microsatellite typing. Sex Transm Infect. 2008;84(2):103-6. doi: 10.1136/sti.2007.025700. [PubMed: 17971371].

3. Moreira D, Paula CR. Vulvovaginal candidiasis. Int J Gynaecol Obstet. 2006;92(3):266-7. doi:10.1016/j.ijgo.2005.12.007. [PubMed:16434042].

4. Abi-Said D, Anaissie E, Uzun O, Raad I, Pinzcowski H, Vartivarian S. The epidemiology of hematogenous candidiasis caused by different Candida species. Clin Infect Dis. 1997;24(6):1122-8. [PubMed: 9195068].

5. Pfaller MA, Houston A, Coffmann S. Application of CHROMagar Candida for rapid screening of clinical specimens for Candida albicans, Candida tropicalis, Candida krusei, and Candida (Torulopsis) glabrata. J Clin Microbiol. 1996;34(1):58-61. [PubMed: 8748273].

6. Novikova N, Rodrigues A, Mardh PA. Can the diagnosis of recurrent vulvovaginal candidosis be improved by use of vaginal lavage samples and cultures on chromogenic agar? Infect Dis Obstet Gynecol. 2002;10(2):89-92. doi: 10.1155/S1064744902000078. [PubMed: 12530485].

7. Sanglard D, Odds FC. Resistance of Candida species to antifungal agents: molecular mechanisms and clinical consequences. Lancet Infect Dis. 2002;2(2):73-85. [PubMed: 11901654].

8. Novak E, Berek JS. Berek \& Novak's gynecology. Lippincott Williams \& Wilkins; 2007.

9. Fatahinia M, Halvaeezadeh M, Rezaei-Matehkolaei A. Comparison of enzymatic activities in different Candida species isolated from women with vulvovaginitis. J Mycol Med. 2017;27(2):188-94. doi: 10.1016/j.mycmed.2017.01.009. [PubMed: 28236530].

10. Gross NT, Arias ML, Moraga M, Baddasarow Y, Jarstrand C. Species distribution and susceptibility to azoles of vaginal yeasts isolated prostitutes. Infect Dis Obstet Gynecol. 2007;2007:82412. doi: 10.1155/2007/82412. [PubMed: 18273407].

11. Espinel-Ingroff A. Clinical relevance of antifungal resistance. Infect Dis Clin North Am. 1997;11(4):929-44. [PubMed: 9421708].

12. Pfaller MA, Yu WL. Antifungal susceptibility testing. New technology and clinical applications. Infect Dis Clin North Am. 2001;15(4):1227-61. [PubMed: 11780273].

13. Pfaller MA, Rex JH, Rinaldi MG. Antifungal susceptibility testing: technical advances and potential clinical applications. Clin Infect Dis. 1997;24(5):776-84. [PubMed: 9142769].
14. Sh M, Sj H. Identification of Pathogenic Candida Species: PCRFragment Size Polymorphism (PCR-FSP) Method. Tehran University Med J TUMS Publications. 2008;66(9):639-45.

15. Kwok S, Higuchi R. Avoiding false positives with PCR. Nature. 1989;339(6221):237-8. doi: 10.1038/339237a0. [PubMed: 2716852].

16. Elie CM, Lott TJ, Reiss E, Morrison CJ. Rapid identification of Candida species with species-specific DNA probes. J Clin Microbiol. 1998;36(11):3260-5. [PubMed: 9774576].

17. Didehdar M, Khansarinejad B, Amirrajab N, Shokohi T. Development of a high-resolution melting analysis assay for rapid and highthroughput identification of clinically important dermatophyte species. Mycoses. 2016;59(7):442-9. doi: 10.1111/myc.12492. [PubMed: 26991756].

18. Williams DW, Wilson MJ, Lewis MA, Potts AJ. Identification of Candida species by PCR and restriction fragment length polymorphism analysis of intergenic spacer regions of ribosomal DNA. J Clin Microbiol. 1995;33(9):2476-9. [PubMed: 7494052].

19. Yamada Y, Makimura K, Uchida K, Yamaguchi H, Osumi M. Phylogenetic relationships among medically important yeasts based on sequences of mitochondrial large subunit ribosomal RNA gene. Mycoses. 2004;47(1-2):24-8. [PubMed:14998395].

20. Mirhendi H, Makimura K, Khoramizadeh M, Yamaguchi H. A oneenzyme PCR-RFLP assay for identification of six medically important Candida species. Nihon Ishinkin Gakkai Zasshi. 2006;47(3):225-9. [PubMed: 16940958].

21. White TJ, Bruns T, Lee S, Taylor JW. Amplification and direct sequencing of fungal ribosomal RNA genes for phylogenetics. PCR protocols: $a$ guide to methods and applications. 1990;18(1):315-22.

22. Mohammadi R, Mirhendi H, Rezaei-Matehkolaei A, Ghahri M, Shidfar MR, Jalalizand N, et al. Molecular identification and distribution profile of Candida species isolated from Iranian patients. Med Mycol. 2013;51(6):657-63. doi: 10.3109/13693786.2013.770603. [PubMed: 23470036].

23. Clinical and Laboratory Standards Institute. . Method for antifungal disk diffusion susceptibility testing of yeasts: Approved guideline. Wayne, PA: Clinical and Laboratory Standards Institute; 2004.

24. Mohammadi R, Nazeri M, Mesdaghinia E, Mirhendi SH. Identification of candida species among patients with vulvovaginal candidiasis in Kashan by PCR-RFLP method.J Isfahan Med School. ;29(165).

25. Hedayati MT, Taheri Z, Galinimoghadam T, Aghili SR, Yazdani Cherati J, Mosayebi E. Isolation of different species of Candida in patients with vulvovaginal candidiasis from sari, iran. Jundishapur J Microbiol. 2015;8(4):e15992. doi: 10.5812/jjm.8(4)2015.15992. [PubMed: 26034533].

26. Nazeri M, Mesdaghinia E, Moraveji SAR, Atabakhshiyan R, Soleymani F. Prevalence of vulvovaginal candidiasis and frequency of candida species in women. J Mazand Univ Med Sci. ;22(86):255-62.

27. Mahmoudi Rad M, Zafarghandi S, Abbasabadi B, Tavallaee M. The epidemiology of Candida species associated with vulvovaginal candidiasis in an Iranian patient population. Eur J Obstet Gynecol Reprod Biol. 2011;155(2):199-203. doi: 10.1016/j.ejogrb.2010.11.022. [PubMed: 21194828].

28. Moallaei H, Ravansalar H, Namazi MJ, Akaberi A. Study and identification of various species of candida in candidiasis vaginitis in women admitted to mobini hospital in sabzevar, Iran (2007).

29. Darce Bello M, Gonzalez A, Barnabe C, Larrouy G. First characterization of Candida albicans by random amplified polymorphic DNA method in Nicaragua and comparison of the diagnosis methods for vaginal candidiasis in Nicaraguan women. Mem Inst Oswaldo Cruz. 2002;97(7):985-9. [PubMed: 12471425].

30. Cheng G, Yeater KM, Hoyer LL. Cellular and molecular biology of Candida albicans estrogen response. Eukaryot Cell. 2006;5(1):180-91. doi: 10.1128/EC.5.1.180-191.2006. [PubMed: 16400181]. 
31. Salehei Z, Seifi Z, Mahmoudabadi A. Sensitivity of vaginal isolates of Candida to eight antifungal drugs isolated from Ahvaz, Iran. Jundishapur J Microbiol. ;5(4):574-7. doi: 10.5812/jjm.4556.

32. Mohanty S, Xess I, Hasan F, Kapil A, Mittal S, Tolosa JE. Prevalence \& susceptibility to fluconazole of Candida species causing vulvovaginitis. Indian J Med Res. 2007;126(3):216-9. [PubMed:18037716].

33. Nyirjesy P, Seeney SM, Grody MH, Jordan CA, Buckley HR. Chronic fungal vaginitis: the value of cultures. Am J Obstet Gynecol. 1995;173(3 Pt 1):820-3. [PubMed: 7573250$]$.

34. Chong PP, Lee YL, Tan BC, Ng KP. Genetic relatedness of Candida strains isolated from women with vaginal candidiasis in Malaysia. J Med Microbiol. 2003;52(Pt 8):657-66. doi: 10.1099/jmm.0.04973-0. [PubMed: 12867559].

35. Richter SS, Galask RP, Messer SA, Hollis RJ, Diekema DJ, Pfaller MA. Antifungal susceptibilities of Candida species causing vulvovaginitis and epidemiology of recurrent cases. J Clin Microbiol. 2005;43(5):2155-62. doi: 10.1128/JCM.43.5.2155-2162.2005. [PubMed: 15872235].

36. Roudbary M, Roudbarmohammadi S, Bakhshi B, Farhadi Z, Nikoomanesh F. Identification of Candida species isolated from Iranian women with vaginal candidiasis by PCR-RFLP method. Europ J of Experiment Biol. ;3(6):365-9.
37. Shi XY, Yang YP, Zhang Y, Li W, Wang JD, Huang WM, et al. Molecular identification and antifungal susceptibility of 186 Candida isolates from vulvovaginal candidiasis in southern China. J Med Microbiol. 2015;64(Pt 4):390-3. doi:10.1099/jmm.0.000024. [PubMed: 25596116]

38. Mahmoudabadi AZ, Najafyan M, Alidadi M. Clinical study of Candida vaginitis in Ahvaz, Iran and susceptibility of agents to topical antifungal. PakJ Med Sci. ;26(3):607-10.

39. El-Din SS, Reynolds MT, Ashbee HR, Barton RC, Evans EG. An investigation into the pathogenesis of vulvo-vaginal candidosis. Sex Transm Infect. 2001;77(3):179-83. [PubMed: 11402224].

40. Lynch ME, Sobel JD. Comparative in vitro activity of antimycotic agents against pathogenic vaginal yeast isolates. J Med Vet Mycol. 1994;32(4):267-74. [PubMed: 7983571].

41. Ribeiro MA, Dietze R, Paula CR, Da Matta DA, Colombo AL. Susceptibility profile of vaginal yeast isolates from Brazil. Mycopathologia. 2001;151(1):5-10. [PubMed: 11502064].

42. Sobel JD, Wiesenfeld HC, Martens M, Danna P, Hooton TM, Rompalo A, et al. Maintenance fluconazole therapy for recurrent vulvovaginal candidiasis. $N$ Engl J Med. 2004;351(9):876-83. doi: 10.1056/NEJMoa033114. [PubMed: 15329425]. 